\title{
CONSTANT BREADTH CURVES IN THE PLANE
}

\author{
P. C. HAMMER
}

In [4] Professor Sobczyk and I have given a generalized construction for all constant breadth convex curves in the plane based on the outwardly simple line families there introduced and characterized and which we further characterize in [5].

In the $(r, s)$-plane any outwardly simple line family $F$ may be represented by

$$
r \sin \theta-s \cos \theta-p(\theta)=0
$$

where $p(\theta+\pi)=-p(\theta)$ and $p(\theta)$ satisfies a Lipschitz condition (cf. [5]).

In this note we give an analytical representation of constant breadth planar curves. Let $u(\theta)$ be the unit vector $(\cos \theta, \sin \theta)$. Let the vector $x=x(\theta)$ be defined by

$$
\mathbf{x}=-p(\theta) \mathbf{u}(\theta)+\left(c-\int_{0}^{\theta} p(\theta) d \theta\right) \mathbf{u}(\theta)
$$

where $c$ is a real number greater than the $\sup _{\theta}\left[D p(\theta)+\int_{0}^{\theta} p(\theta) d \theta\right]$ in which $D p(\theta)$ represents any of the possible limits of sequences of difference quotients of $p$ at $\theta$.

ThEOREM. Under the conditions stated, (2) represents a constant breadth convex curve which is orthogonal to the line family (1) and has a continuously turning tangent line at each point. Letting $c$ approach $\sup _{\theta}\left[D p(\theta)+\int_{0}^{0} p(\theta) d \theta\right]$ from above, a limiting constant breadth convex curve (or a point if $F$ is a pencil) is obtained. With this inclusion (2) represents all constant breadth convex curves in the plane.

Proof. It has been previously shown [4] that there exists a unique orthogonal trajectory to a planar outwardly simple line family $F$, through a point sufficiently far from the origin. Let $\theta$ be given and since the difference quotients of $p$ are bounded, let $\left\{h_{n}\right\}$ represent any null sequence such that the difference quotients of $p$ at $\theta$ with differences $\left\{h_{n}\right\}$ converge to a number $D p(\theta)$. Then a simple calculation shows that $D \mathbf{x}(\theta)=\left[c-D p(\theta)-\int_{0}^{\theta} p(\theta) d \theta\right] \mathbf{u}^{\prime}(\theta)$ since $\boldsymbol{u}(\theta)=-\boldsymbol{u}^{\prime \prime}(\theta)$. Now for $c>\sup _{\theta}\left[D p(\theta)+\int_{0}^{\theta} p(\theta) d \theta\right]$ we have that $D \mathbf{x}(\theta)$ is parallel to $u^{\prime}(\theta)$ and has the same sense. Hence the curve given by (2) is orthogonal to the lines in $F$ and (cf. [4]) this curve is a constant diameter

Received by the editors November 5, 1953. 
convex curve. The rest of the theorem then follows readily from the geometrical consideration in [4] based on results in [3]. Q.E.D.

Note that if the number $c$ is taken so that $c-D p(\theta)-\int_{0}^{\theta} p(\theta) d \theta$ changes signs, then we get nonconvex constant breadth closed curves. If $c$ is taken so that the quantity above is always negative, then the same convex constant breadth curves are obtained. The breadth of a curve is $\left|2 c-\int_{0}^{\pi} p(\theta) d \theta\right|$. If we let $p(\theta)$ still satisfy the Lipschitz condition but have other periodicities, we may obtain other closed nonconvex curves of constant breadth. For example if $p(\theta+3 \pi)=-p(\theta)$ we would have a constant breadth curve from (2) of breadth $\left|2 c-\int_{0}^{3 \pi} p(\theta) d \theta\right|$.

Now, if one lets $h(\theta)=\left(c-\int_{0}^{\theta} p d \theta\right)$, then formula (2) becomes

$$
\mathbf{x}(\theta)=h^{\prime}(\theta) \mathbf{u}^{\prime}(\theta)+h(\theta) \mathbf{u}(\theta)
$$

where $h(\theta)$ is the support function of the constant breadth curve. In the various other papers of which we are aware containing variants of (3) the differentiability conditions imposed are unnecessarily stringent. The result here, based on the vantage point of outwardly simple line families, seems to be first to establish necessary as well as sufficient conditions. The monograph [1] of Bonnesen and Fenchel contains references to a large number of papers concerned with constant breadth curves. Basically equation (3) is due to Euler [2].

\section{REFERENCES}

1. T. Bonnesen and W. Fenchel, Konvexe Koerper, New York, 1948 (Reprint).

2. L. Euler, De curvis triangularibus, Acta Academiae Petropolitoneae (1778) pp. 3-30.

3. P. C. Hammer, Convex bodies associated with a convex body, Proc. Amer. Math. Soc. vol. 2 (1951) pp. 781-793.

4. P. C. Hammer and A. Sobczyk, Planar line families. I, Proc. Amer. Math. Soc. vol. 4 (1953) pp. 226-233.

5. —_, Planar line families. II, Proc. Amer. Math. Soc. vol. 4 (1953) pp. 341349.

UNIVERSITY OF WISCONSIN 